\title{
Predicting Translation Performance with Referential Translation Machines
}

\author{
Ergun Biçici \\ orcid.org/0000-0002-2293-2031 \\ bicici.github.com
}

\begin{abstract}
Referential translation machines achieve top performance in both bilingual and monolingual settings without accessing any task or domain specific information or resource. RTMs achieve the 3rd system results for German to English sentence-level prediction of translation quality and the 2nd system results according to root mean squared error. In addition to the new features about substring distances, punctuation tokens, character $n$-grams, and alignment crossings, and additional learning models, we average prediction scores from different models using weights based on their training performance for improved results.
\end{abstract}

\section{Introduction}

Quality estimation task (QET) in WMT17 (Bojar et al., 2017) (QET17) is about prediction of the quality of machine translation output at the sentence- (Task 1), word- (Task 2), and phraselevel (Task 3) in IT and pharmaceutical domains without using reference translations. Prediction of translation performance can help in estimating the effort required for correcting the translations during post-editing by human translators if needed. RTMs are capable to model different domains and tasks while achieving top performance in both monolingual (Biçici and Way, 2015) and bilingual settings (Biçici, 2016b). We develop RTM models for all of the three subtasks of QET17, which include English to German (en-de), and German to English (de-en) translation directions. Task 1 is about predicting HTER (human-targeted translation edit rate) scores (Snover et al., 2006), Task 2 is about binary classification of word-level quality,

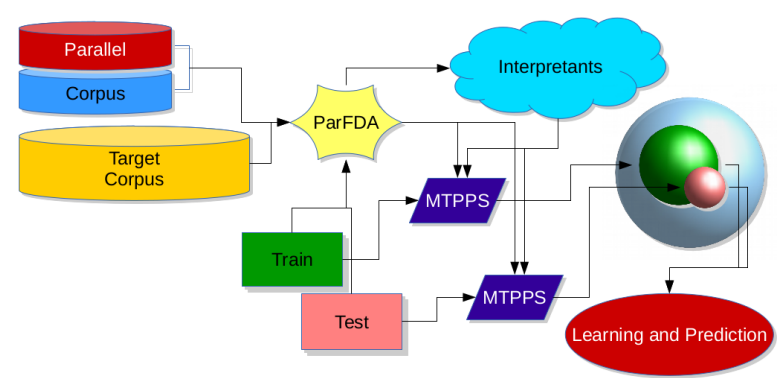

Figure 1: RTM depiction: ParFDA selects interpretants close to the training and test data using parallel corpus in bilingual settings and monolingual corpus in the target language or just the monolingual target corpus in monolingual settings; an MTPPS use interpretants and training data to generate training features and another use interpretants and test data to generate test features in the same feature space; learning and prediction takes place taking these features as input.

and Task 3 is about binary classification of phraselevel quality.

\section{Referential Translation Machines}

Referential translation machine (RTM) models are predict data translation between the instances in the training set and the test set. RTMs use interpretants, data close to the task instances, to derive features measuring the closeness of the test sentences to the training data, the difficulty of translating them, and to identify translation acts between any two data sets for building prediction models. RTMs are applicable in different domains and tasks and in both monolingual and bilingual settings. Figure 1 depicts RTMs and explains the model building process. RTMs use ParFDA (Biçici, 2016a) for instance selection and machine translation performance prediction system (MTPPS) (Biçici and Way, 2015) for generat- 


\begin{tabular}{|c|c|c|c|c|c|c|c|c|c|c|}
\hline Task & Model & DeltaAvg & $r_{P}$ & $r_{S}$ & RMSE & MAE & RAE & MAER & MRAER & Rank \\
\hline on & MIX 4 & 8.64 & 0.4544 & 0.4768 & 0.1707 & 0.1296 & 0.8483 & 0.7594 & 0.7962 & 9 \\
\hline \multirow{3}{*}{ Task 1} & PLS GBR & 8.22 & 0.4302 & 0.4518 & 0.1727 & 0.1311 & 0.8586 & 0.7769 & 0.8099 & 10 \\
\hline & MIX 4 & 8.94 & 0.6004 & 0.5704 & 0.1566 & 0.1085 & 0.7034 & 0.7201 & 0.6921 & 4 \\
\hline & TREE & 9.18 & 0.5845 & 0.5729 & 0.158 & 0.1186 & 0.7685 & 0.9013 & 0.7627 & 5 \\
\hline
\end{tabular}

Table 1: Task 1 test results of the top 2 individual RTM models. RTM becomes the 2nd system according to RMSE and 3rd system in de-en and 6th system in en-de. $r_{P}$ is Pearson's correlation and $r_{S}$ is Spearman's correlation.

\begin{tabular}{l|cc|cc} 
& & & \multicolumn{2}{|c}{ RTM Interpretants } \\
Task & Train & Test & Training & LM \\
\hline Task 1, 2, 3 (en-de) & 24000 & 2000 & $1.1 \mathrm{M}$ & $17.6 \mathrm{M}$ \\
Task 1, 2, 3 (de-en) & 26000 & 2000 & $1.1 \mathrm{M}$ & $17.6 \mathrm{M}$ \\
\hline
\end{tabular}

Table 2: Number of instances used as interpretants by the RTM models.

ing features where the total number of features becomes 514, increasing depending on the order of $n$-grams used and we used up to 5 -grams for translation features and 7-grams for language model (LM) at QET17.

We use ridge regression (RR), k-nearest neighors (KNN), support vector regression (SVR), AdaBoost (Freund and Schapire, 1997), and extremely randomized trees (TREE) (Geurts et al., 2006) as learning models in combination with feature selection (FS) (Guyon et al., 2002) and partial least squares (PLS) (Wold et al., 1984). We use scikit-learn ${ }^{1}$ for most of these models. The following parameters are optimized: $\lambda$ for $\mathrm{RR}, \mathrm{k}$ for KNN, $\gamma, \mathrm{C}$, and $\epsilon$ for SVR, minimum number of samples for leaf nodes and for splitting an internal node for TREE, the number of features for FS, and the number of dimensions for PLS. For AdaBoost, we do not optimize but use exponential loss and 500 estimators like we use also with the TREE model. We use grid search for SVR. Evaluation metrics we use are Pearson's correlation $(r)$, mean absolute error (MAE), relative absolute error (RAE), MAER (mean absolute error relative), and MRAER (mean relative absolute error relative) (Biçici and Way, 2015). DeltaAvg (CallisonBurch et al., 2012) calculates the average quality difference between the top $n-1$ quartiles and the overall quality for the test set. Official evaluation metrics include $r$, MAE, and DeltaAvg.

We improved RTM models (Biçici, 2016b) with additional features:

- normalized Levenshtein distance between the

\footnotetext{
${ }^{1}$ http://scikit-learn.org/
}

source sentence and its translation and their longest common prefix, suffix, and substring (Tian et al., 2017) normalized by the minimum length of the compared sentences.

- number of tokens about punctuation in the source sentence and the translation (Kozlova et al., 2016) and the cosine between them.

- modified $\mathrm{CHR}_{3}$ (Popović, 2015) to compute character $n$-grams split by word boundary space with $n \in[3,7]$ whereas the $F_{1}$ (Biçici, 2011) we already use compute with word $n$-grams up to $n=5$.

- proportion of alignments that cross $(\mathbb{A})$ the link (Sagemo and Stymne, 2016) of any other alignments:

$$
\sqrt{\frac{0.5 \times|a \times A|}{|A|}}
$$

- word alignment correspondence features (Sagemo and Stymne, 2016).

- additional learning models including KNN, AdaBoost, and gradient boosting regressor (GBR) (Tian et al., 2017; Hastie et al., 2009).

We also use prediction averaging (Biçici, 2017), where the performance on the training set is used to obtain weighted average of the top $k$ predictions, $\hat{y}$ with evaluation metrics indexed by $j \in J$ :

$$
\begin{array}{lll}
\hat{\boldsymbol{y}}_{\mu_{k}}=\frac{1}{k} \sum_{i=1}^{k} \hat{\boldsymbol{y}}_{i} & \text { MEAN } \\
\hat{\boldsymbol{y}}_{j, w_{k}^{j}}=\frac{1}{\sum_{i=1}^{k} \frac{1}{w_{j, i}} \sum_{i=1}^{k} \frac{1}{w_{j, i}} \hat{\boldsymbol{y}}_{i}} & \\
\hat{\boldsymbol{y}}_{k} & =\frac{1}{|J|} \sum_{j \in J} \hat{\boldsymbol{y}}_{j, w_{k}^{j}} & \text { MIX }
\end{array}
$$

MAER is used to select the predictions and weights are inverted to decrease error.

We use Global Linear Models (GLM) (Collins, 2002) with dynamic learning (GLMd) (Biçici, 


\begin{tabular}{|c|c|c|c|}
\hline Model & splits & $\%$ error & weights \\
\hline GLMd & 4 & 0.0773 & \multirow{12}{*}[0.5,2]{} \\
\hline${ }^{\text {en-de GLMd }}$ & 5 & 0.0668 & \\
\hline word $\frac{\text { GLMd }}{d e_{-} \text {en }}$ & 4 & 0.0468 & \\
\hline de-en GLMd & 5 & 0.0469 & \\
\hline $2017-$ GLMd $_{\text {en-de }}$ & 4 & 0.0068 & \\
\hline en-de GLMd & 5 & 0.0059 & \\
\hline \multirow{2}{*}{ de-en } & 4 & 0.0129 & \\
\hline & 5 & 0.0125 & \\
\hline \multirow{2}{*}{ word en-de $\begin{array}{l}\text { GLMd } \\
\text { GLMd }\end{array}$} & 4 & 0.0688 & \\
\hline & 5 & 0.0757 & \\
\hline \multirow{2}{*}{ phrase en-de $\begin{array}{l}\text { GLMd } \\
\text { GLMd }\end{array}$} & 4 & 0.0051 & \\
\hline & 5 & 0.0051 & \\
\hline
\end{tabular}

Table 3: RTM Task 2 training results where GLMd parallelized over 4 splits is referred as GLMd s4 and GLMd with 5 splits as GLMd s5.

\begin{tabular}{|c|c|c|c|c|c|}
\hline & Moc & & $F_{1} \mathrm{BAD}$ & $F_{1} \mathrm{OK}$ & $\mathrm{w} F_{1}$ \\
\hline \multirow{4}{*}{ Word } & \multirow{2}{*}{ en-de } & GLMd s4 & 0.318 & 0.8844 & 0.2813 \\
\hline & & GLMd s5 & 0.36 & 0.8778 & 0.3158 \\
\hline & \multirow{2}{*}{ de-en } & GLMd s4 & 0.3363 & 0.9386 & 0.3157 \\
\hline & & GLMd s5 & 0.3381 & 0.9395 & 0.3176 \\
\hline \multirow{4}{*}{ Phrase } & \multirow{2}{*}{ en-de } & GLMd s4 & 0.4043 & 0.8079 & 0.3283 \\
\hline & & GLMd s5 & 0.4114 & 0.8079 & 0.3323 \\
\hline & \multirow{2}{*}{ de-en } & GLMd s4 & 0.2472 & 0.9073 & 0.2242 \\
\hline & & GLMd s5 & 0.3598 & 0.8884 & 0.3197 \\
\hline
\end{tabular}

Table 4: RTM Task 2 results on the test set after the challenge. w $F_{1}$ is average weighted $F_{1}$ score.

2016b) for word- and phrase-level translation performance prediction. GLMd uses weights in a range $[a, b]$ to update the learning rate dynamically according to the error rate.

\section{Results}

Table 2 lists the number of sentences in the training and test sets for each task and the number of instances used as interpretants in the RTM models ( $\mathrm{M}$ for million). We tokenize and truecase all of the corpora using Moses's (Koehn et al., 2007) processing tools. ${ }^{2}$ LMs are built using KENLM (Heafield et al., 2013).

\subsection{QET 2017 Results}

The results on the Task 1 test set are listed in Table 1. ${ }^{3}$ For Task 2 and Task 3, we list the results

\footnotetext{
${ }^{2}$ https://github.com/moses-smt/ mosesdecoder/tree/master/scripts

${ }^{3}$ We calculate $r_{S}$ using scipy. stats.
}

tation on the training sets in Table 3 and on the test set in Table 4. The results we obtained in the challenge are similar. Ranks for Task 1 are out of 14 submissions and 9 systems. Top RTM models that competed in Task 1 were MIX 4, which combines top 4 predictions, PLS GBR, and TREE. RTM becomes the 2 nd system according to RMSE and 3rd system in de-en and 6th system in en-de.

\subsection{Recomputing QET 2016 Results}

QET17 also compares results on QET16 test sets. QET16 test set domain was different than the domain of QET17, overlapping on the IT domain. We use the RTM models built for QET17 to obtain results on the QET16 test sets, which is categorized as transductive transfer learning. ${ }^{4}$ Transfer learning attempt to re-use and transfer knowledge from models developed in different domains or for different tasks such as using models developed for handwritten digit recognition for handwritten character recognition (Guyon et al., 2012). The results are in Table 5 for Task 1 , which does not show improvement, and in Table 7, which show improvements with RTM models built for QET17.

\subsection{Comparison with Previous Results}

We compare the difficulty of tasks according to MRAER levels achieved. In Table 6, we list the RTM test results when predicting sentence-level HTER in 2013-2017. Compared with QET16, we observe improvements in MRAER and both MAE and RAE are improved when QET17 is compared with others.

\section{Conclusion}

Referential translation machines achieve top performance in automatic, accurate, and language independent prediction of translation performance and achieve to become the 2nd system according to RMSE when predicting the translation performance from German to English. RTMs pioneer a language independent approach for predicting translation performance and remove the need to access any task or domain specific information or resource.

\footnotetext{
${ }^{4}$ Www youtube. com/wat ch? $\mathrm{v}=9 \mathrm{ChVn} 3 \mathrm{xVNDI}$; we use the RTM models for the same task in different domains.
} 


\begin{tabular}{ll|lllllll}
\multicolumn{2}{c|}{ Model } & DeltaAvg & $r$ & MAE & RMSE & RAE & MAER & MRAER \\
\hline \multirow{2}{*}{2017} & ST TREE & 5.14 & 0.2052 & 0.1456 & 0.1875 & 0.9634 & 0.8844 & 0.8666 \\
& PLS GBR & 3.71 & 0.1875 & 0.1474 & 0.1914 & 0.9755 & 0.8706 & 0.8966 \\
\hline \multirow{2}{*}{2016} & SVR & 6.38 & 0.3581 & 0.1359 & 0.1806 & 0.8992 & 0.7509 & 0.8567 \\
& FS SVR & 6.66 & 0.3764 & 0.1346 & 0.1781 & 0.8905 & 0.7537 & 0.8388 \\
\hline
\end{tabular}

Table 5: QET16 Task 1 results are not improved with QET17 Task 1 RTM models.

\begin{tabular}{lll|lllll}
\multirow{2}{*}{ Task } & \multicolumn{2}{c|}{ Translation Model } & $r$ & MAE & RAE & MAER & MRAER \\
\hline \multirow{2}{*}{ QET17 Task 1 HTER } & en-de & MIX 4 & 0.4544 & 0.1296 & 0.8483 & 0.7594 & 0.7962 \\
\cline { 2 - 7 } & de-en & MIX 4 & 0.6004 & 0.1085 & 0.7034 & 0.7201 & 0.6921 \\
\hline QET16 Task 1 HTER & en-de & FS SVR & 0.3764 & 0.1346 & 0.8905 & 0.7537 & 0.8388 \\
\hline QET15 Task 1 HTER & en-es & FS+PLS SVR & 0.349 & 0.1335 & 0.903 & 0.8284 & 0.8353 \\
\hline QET14 Task 1.2 HTER & en-es & SVR & 0.5499 & 0.134 & 0.8532 & 0.7727 & 0.8758 \\
\hline QET13 Task 1.1 HTER & en-es & PLS-SVR & 0.5596 & 0.1326 & 0.8849 & 2.3738 & 1.6428 \\
\hline
\end{tabular}

Table 6: Test performance of the top RTM results when predicting sentence-level HTER in 2013-2017.

\begin{tabular}{ll|ccc}
\multicolumn{1}{c|}{ Model } & w $F_{1}$ & $F_{1}$ OK & $F_{1}$ BAD \\
\hline \multirow{2}{*}{ Word } & GLMd s4 & 0.2857 & 0.8775 & 0.3256 \\
& GLMd s5 & 0.3053 & 0.8653 & 0.3528 \\
\hline \multirow{2}{*}{ Phrase } & GLMd s4 & 0.3421 & $\mathbf{0 . 8 1 9 2}$ & 0.4176 \\
& GLMd s5 & 0.3504 & $\mathbf{0 . 8 1 7}$ & 0.4289 \\
\hline \multirow{2}{*}{ Word } & GLMd s4 & 0.2725 & $\mathbf{0 . 8 8 8 4}$ & 0.3068 \\
& GLMd s5 & 0.3081 & 0.8820 & 0.3494 \\
\hline \multirow{2}{*}{ Phrase } & GLMd s4 & 0.3070 & $\mathbf{0 . 8 1 4 5}$ & 0.3770 \\
& GLMd s5 & 0.3274 & $\mathbf{0 . 8 0 1 6}$ & 0.4084 \\
\hline
\end{tabular}

Table 7: QET16 Task 2 and Task 2p results show improvement.

\section{References}

Ergun Biçici. 2011. The Regression Model of Machine Translation. Ph.D. thesis, Koç University. Supervisor: Deniz Yuret.

Ergun Biçici. 2016a. ParFDA for instance selection for statistical machine translation. In Proc. of the First Conference on Statistical Machine Translation (WMT16). Association for Computational Linguistics, Berlin, Germany. http://aclanthology.info/papers/parfda-for-instanceselection-for-statistical-machine-translation.

Ergun Biçici. 2016b. Referential translation machines for predicting translation performance. In Proc. of the First Conference on Statistical Machine Translation (WMT16). Association for Computational Linguistics, Berlin, Germany. http://aclanthology.info/papers/referentialtranslation-machines-for-predicting-translation-per formance.

Ergun Biçici. 2017. RTM at SemEval-2017 task 1: Referential translation machines for predicting semantic similarity. In Proc. of the 11th International Workshop on Semantic
Evaluation (SemEval-2017). Association for Computational Linguistics, Vancouver, Canada, pages 194-198. http://nlp.arizona.edu/SemEval2017/pdf/SemEval030.pdf.

Ergun Biçici and Andy Way. 2015. Referential translation machines for predicting semantic similarity. Language Resources and Evaluation pages 1-27. https://doi.org/10.1007/s10579-015-9322-7.

Ondrej Bojar, Christian Buck, Rajen Chatterjee, Christian Federmann, Barry Haddow, Matthias Huck, Jimeno Antonio Yepes, Julia Kreutzer, Varvara Logacheva, Aurelie Neveol, Mariana Neves, Philipp Koehn, Christof Monz, Matteo Negri, Matt Post, Stefan Riezler, Artem Sokolov, Lucia Specia, Karin Verspoor, and Marco Turchi. 2017. Proc. of the second conference on Machine Translation. In Proc. of the Second Conference on Machine Translation. Association for Computational Linguistics, Copenhagen, Denmark.

Chris Callison-Burch, Philipp Koehn, Christof Monz, Matt Post, Radu Soricut, and Lucia Specia. 2012. Findings of the 2012 workshop on statistical machine translation. In Proc. of the Seventh Workshop on Statistical Machine Translation. Montréal, Canada, pages 10-51.

Michael Collins. 2002. Discriminative training methods for hidden markov models: theory and experiments with perceptron algorithms. In Proc. of the ACL-02 conference on Empirical methods in natural language processing - Volume 10. Stroudsburg, PA, USA, EMNLP '02, pages 1-8. https://doi.org/10.3115/1118693.1118694.

Yoav Freund and Robert E Schapire. 1997. A decision-theoretic generalization of on-line learning and an application to boosting. Journal of Computer and System Sciences 55(1):119-139. https://doi.org/10.1006/jcss.1997.1504. 
Pierre Geurts, Damien Ernst, and Louis Wehenkel. 2006. Extremely randomized trees. Machine Learning 63(1):3-42.

Isabelle Guyon, Gideon Dror, Vincent Lemaire, Graham W. Taylor, and Daniel L. Silver, editors. 2012. Unsupervised and Transfer Learning - Workshop held at ICML 2011, Bellevue, Washington, USA, July 2, 2011, volume 27 of JMLR Proceedings. JMLR.org. http://clopinet.com/ul.

Isabelle Guyon, Jason Weston, Stephen Barnhill, and Vladimir Vapnik. 2002. Gene selection for cancer classification using support vector machines. Machine Learning 46(1-3):389-422. https://doi.org/10.1023/A:1012487302797.

Trevor Hastie, Robert Tibshirani, and Jerome Friedman. 2009. The Elements of Statistical Learning: Data Mining, Inference and Prediction. SpringerVerlag, 2nd edition.

Kenneth Heafield, Ivan Pouzyrevsky, Jonathan H. Clark, and Philipp Koehn. 2013. Scalable modified Kneser-Ney language model estimation. In Proc. of the 51st Annual Meeting of the Association for Computational Linguistics. Sofia, Bulgaria, pages 690 696.

Philipp Koehn, Hieu Hoang, Alexandra Birch, Chris Callison-Burch, Marcello Federico, Nicola Bertoldi, Brooke Cowan, Wade Shen, Christine Moran, Richard Zens, Chris Dyer, Ondrej Bojar, Alexandra Constantin, and Evan Herbst. 2007. Moses: Open source toolkit for statistical machine translation. In Proc. of the 45th Annual Meeting of the Association for Computational Linguistics Companion Volume Proc. of the Demo and Poster Sessions. Association for Computational Linguistics, pages 177-180. aclweb.org/anthology/P07-2045.

Anna Kozlova, Mariya Shmatova, and Anton Frolov. 2016. Ysda participation in the wmt'16 quality estimation shared task. In Proceedings of the First Conference on Machine Translation. Association for Computational Linguistics, Berlin, Germany, pages 793-799. http://www.aclweb.org/anthology/W/W16/W162385 .

Maja Popović. 2015. chrf: character n-gram fscore for automatic mt evaluation. In Proceedings of the Tenth Workshop on Statistical Machine Translation. Association for Computational Linguistics, Lisbon, Portugal, pages 392-395. http://aclweb.org/anthology/W15-3049.

Oscar Sagemo and Sara Stymne. 2016. The uu submission to the machine translation quality estimation task. In Proceedings of the First Conference on Machine Translation. Association for Computational Linguistics, Berlin, Germany, pages 825-830. http://www.aclweb.org/anthology/W/W16/W162390.
Matthew Snover, Bonnie Dorr, Richard Schwartz, Linnea Micciulla, and John Makhoul. 2006. A Study of Translation Edit Rate with Targeted Human Annotation. In Proc. of Association for Machine Translation in the Americas,

Junfeng Tian, Zhiheng Zhou, Man Lan, and Yuanbin Wu. 2017. Ecnu at semeval-2017 task 1: Leverage kernel-based traditional nlp features and neural networks to build a universal model for multilingual and cross-lingual semantic textual similarity. In Proceedings of the 11th International Workshop on Semantic Evaluation (SemEval-2017). Association for Computational Linguistics, Vancouver, Canada, pages 182188. http://www.aclweb.org/anthology/S17-2028.

S. Wold, A. Ruhe, H. Wold, and W. J. III Dunn. 1984. The collinearity problem in linear regression. the partial least squares (pls) approach to generalized inverses. SIAM Journal on Scientific and Statistical Computing 5:735-743. 